\title{
Variabilités spatiales et temporelles de la qualité physico-chimique et des invertébrés pélagiques des eaux de la retenue hydroélectrique de Petit Saut (Guyane française). The Petit Saut hydroelectric dam (French Guiana) : temporal and spatial variability of the water quality and pelagic invertebrates
}

\author{
V. Horeau, S. Richard, R. Vigouroux, L. Guillemet et P. Cerdan
}

Volume 18, numéro hors-série, 2005

URI : https://id.erudit.org/iderudit/705579ar

DOI : https://doi.org/10.7202/705579ar

\section{Aller au sommaire du numéro}

\section{Éditeur(s)}

Université du Québec - INRS-Eau, Terre et Environnement (INRS-ETE)

\section{ISSN}

0992-7158 (imprimé)

1718-8598 (numérique)

\section{Découvrir la revue}

Citer cet article

Horeau, V., Richard, S., Vigouroux, R., Guillemet, L. \& Cerdan, P. (2005). Variabilités spatiales et temporelles de la qualité physico-chimique et des invertébrés pélagiques des eaux de la retenue hydroélectrique de Petit Saut (Guyane française). Revue des sciences de l'eau / Journal of Water Science, 18 , 109-126. https://doi.org/10.7202/705579ar

\section{Résumé de l'article}

Le bassin versant du fleuve Sinnamary est situé en Guyane française, au nord du continent sud américain, en zone néo-tropicale. Il s'étend entre 4 et $5^{\circ} \mathrm{de}$ latitude Nord et entre $52^{\circ} 50^{\prime}$ et $53^{\circ} 30^{\prime}$ de longitude Ouest. En 1994, la mise en eau du barrage hydroélectrique construit sur le site de Petit Saut occasionne l'ennoiement de $365 \mathrm{~km} 2$ de forêt primaire. Le remplissage s'effectue en 18 mois, et la demande en oxygène dissous nécessaire à la dégradation de la matière organique est telle qu'en quelques jours la masse d'eau se stratifie en un épilimnion oxygéné et un hypolimnion anoxique. Représentant quelques centimètres en 1994, l'épilimnion s'épaissit progressivement jusqu'en 1998. Depuis, cette progression s'est nettement ralentie et l'épilimnion oscille autour d'une valeur moyenne de 5 à 6 mètres. Il abrite la plus grande partie du zooplancton pélagique. L'hypolimnion est anoxique et riche en éléments réduits. Le zooplancton s'est rapidement installé avec des rotifères, des cladocères et des copépodes. Des ostracodes et des Chaoboridae y sont désormais associés. La retenue présente une zonation longitudinale, de la tête de la retenue vers la queue, aussi bien en termes de densités que de composition des peuplements. De même, il existe une zonation transversale, notamment marquée par un gradient croissant de la conductivité de l'axe vers les berges. Ces ressources endogènes ainsi que des apports complémentaires (végétaux, invertébrés terrestres, détritus...) sont utilisées par la faune ichtyque qui a su s'adapter au changement du milieu. Le temps de rétention des eaux qui fluctue saisonnièrement en fonction des pluies semble être le facteur prépondérant de variabilité pour la qualité physico-chimique des eaux et les communautés biologiques. 


\title{
Variabilités spatiales et temporelles de la qualité physico-chimique et des invertébrés pélagiques des eaux de la retenue hydroélectrique de Petit Saut (Guyane française)
}

\author{
The Petit Saut hydroelectric dam (French Guiana): \\ temporal and spatial variability of the water quality \\ and pelagic invertebrates
}

\section{HOREAU ${ }^{\star *}$, S. RICHARD², R. VIGOUROUX², L. GUILLEMET², P. CERDAN²}

Reçu le 5 décembre 2003, accepté le 27 octobre 2004**.

SUMMARY

The Sinnamary River is located in a neotropical zone, in French Guiana, in the northern part of South America. Its flood basin extends between $4^{\circ}$ and $5^{\circ} \mathrm{N}$ latitude and $52^{\circ} 50^{\prime}$ and $53^{\circ} 30^{\prime} \mathrm{W}$ longitude. The river is found in an equatorial climate, and is influenced by its proximity to the ocean. The temperature varies little, with an average of $25^{\circ} \mathrm{C}$ for the least hot month (January) and $27^{\circ} \mathrm{C}$ for the hottest month (October). The humidity is always high and averages more than $90 \%$. The Sinnamary River bed is broad (>50 m), and runs from south to north for approximately $240 \mathrm{~km}$ with a very weak slope $(0.0003 \%)$. Petit Saut, the site of the hydroelectric dam, is located approximately $60 \mathrm{~km}$ from the mouth of the river, with a flood basin area of $5927 \mathrm{~km}^{2}$ and an average flow rate $260 \mathrm{~m}^{3} / \mathrm{s}$.

The construction of the hydroelectric dam at Petit Saut in 1994 resulted in the flooding of $365 \mathrm{~km}^{2}$ of primary forest in a neotropical zone (the average depth of the reservoir is $11 \mathrm{~m}$ ). The dam reservoir was filled over a period of 18 months and the amount of dissolved oxygen necessary for the organic matter to decompose was such that, in a matter of days, the body of water became stratified into an oxygenated epilimnion and an anoxic hypolimnion. Only centimeters thick in 1994, by 1998 the epilimnion had progressively expanded. Since that time it has varied between an average thickness of 5 to $6 \mathrm{~m}$ and is home to various biological communities. The hypolimnion is anoxic and rich in reduced compounds (nitrogen and phosphorus minerals, dissolved organic matter, particles, metals and gas). The products of the degradation of the flooded organic matter, which still prevail at the bottom,

1. Laboratoire Environnement de Petit Saut, HYDREC0, BP 823, 97388 Kourou Cedex, France, tél. : 05943273 01, fax : 0594322129.

2. Laboratoire Environnement de Petit Saut, HYDRECO, BP 823, 97388 Kourou Cedex, France.

* Correspondance: horeau.veronique@wanadoo.fr

** Les commentaires seront reçus jusqu’au 31 mai 2006. 
are gradually relayed towards surface by phenomena of dilution, hydrolysis and oxidation.

The river fauna disappeared from the reservoir and was replaced after a few weeks by various pelagic fauna. The zooplanktonic communities, primarily confined to the epilimnion, were quickly settled with rotifera, cladocera and copepoda. Initially dominated by detritivores (Bosminidae) and Cyclopidae from February to May 1994, the settlements diversified with phytophagous zooplankton (Calanidae and Daphniidae) when the phytoplankton developed. This procession was quickly accompanied by Sididae, which has dominated the cladocera since mid-1996 and by ostracoda, which were particularly abundant from 1995 to 1997 . Carnivores such as Chaoboridae larvae were also present in Petit Saut reservoir. In contrast to the other zooplankton species, Chaoboridae larvae occupy the entire water column. As a result of great quantities of organic matter available at the time the reservoir was filled, the biomasses were initially large and have gradually decreased with decreasing nutrient concentrations.

The reservoir was not homogeneous in terms of zooplankton density, with a longitudinal zonation from the dam towards the upstream tail. In the tail, fauna were identical to those of the river. In the lake zone, phytophagous Calanidae dominated. In the intermediate zone, known as the zone of transition, the zooplankton biomass normalized to the volume of oxygenated water was less important than in the lake zone. Cyclopidae, well represented at the time of the reservoir colonization, dominated the communities in the transition zone. This distribution of zooplankton was likely connected with the dissolved oxygen concentrations, as well as with the quantities of phytoplankton and bacterio-chlorophyll. Similarly, there was a transverse zonation, marked by an increase in conductivity towards the reservoir banks. The density of the communities varied seasonally. The highest biomasses were measured along the central axis at the time of high waters and close to the banks at the time of low waters.

The time of water retention, which fluctuates according to the rainy season, seemed to be the factor dominating the physicochemical water quality variability and the biological communities. The rainy season is characterized by the dilution of various elements (e.g., conductivity is reduced). Conversely, during the dry season, the elements concentrate again in the water mass. Nevertheless, the peaks of pelagic invertebrate density were observed during times of high waters, when the hydraulic conditions favour the exchanges between the hypolimnion (anoxic and rich in nutritive elements) and the epilimnion (oxygenated and very low in nutrients).

These endogenous resources, as well as complementary contributions (vegetation, invertebrate, terrestrial, refuse), are used by the fish fauna, which adapted to the change in their environment. Predation contributes to the seasonal evolution of the zooplankton. Predation pressure was more important in the dry season than in the rainy season.

Keywords: Physico-chemistry water quality, pelagic invertebrates, Sinnamary river, Petit Saut hydroelectric dam, French Guiana.

Le bassin versant du fleuve Sinnamary est situé en Guyane française, au nord du continent sud-américain, en zone néo-tropicale. Il s'étend entre 4 et $5^{\circ}$ de latitude Nord et entre $52^{\circ} 50^{\prime}$ et $53^{\circ} 30^{\prime}$ de longitude Ouest. En 1994, la mise en eau du barrage hydroélectrique construit sur le site de Petit Saut occasionne l'ennoiement de $365 \mathbf{~ k m}^{2}$ de forêt primaire. Le remplissage s'effectue en 18 mois, et la demande en oxygène dissous nécessaire à la dégradation de la matière organique est telle qu'en quelques jours la masse d'eau se 
stratifie en un épilimnion oxygéné et un hypolimnion anoxique. Représentant quelques centimètres en 1994, l'épilimnion s'épaissit progressivement jusqu'en 1998. Depuis, cette progression s'est nettement ralentie et l'épilimnion oscille autour d'une valeur moyenne de 5 à 6 mètres. Il abrite la plus grande partie du zooplancton pélagique. L'hypolimnion est anoxique et riche en éléments réduits. Le zooplancton s'est rapidement installé avec des rotifères, des cladocères et des copépodes. Des ostracodes et des Chaoboridae y sont désormais associés. La retenue présente une zonation longitudinale, de la tête de la retenue vers la queue, aussi bien en termes de densités que de composition des peuplements. De même, il existe une zonation transversale, notamment marquée par un gradient croissant de la conductivité de l'axe vers les berges. Ces ressources endogènes ainsi que des apports complémentaires (végétaux, invertébrés terrestres, détritus...) sont utilisées par la faune ichtyque qui a su s'adapter au changement du milieu. Le temps de rétention des eaux qui fluctue saisonnièrement en fonction des pluies semble être le facteur prépondérant de variabilité pour la qualité physico-chimique des eaux et les communautés biologiques.

Mots-clés : qualité physico-chimique, invertébrés pélagiques, fleuve Sinnamary, Petit Saut, barrage hydroélectrique, Guyane française.

\section{1 - INTRODUCTION}

Le fleuve Sinnamary est situé en Guyane française, au nord du continent sud-américain. Son bassin versant de $5927 \mathrm{~km}^{2}$ à Petit Saut (site du barrage) s'étend entre 4 et $5^{\circ}$ de latitude Nord et entre $52^{\circ} 50^{\prime}$ et $53^{\circ} 30^{\prime}$ de longitude Ouest. Très peu d'études concernant la qualité physico-chimique des eaux et de certains invertébrés aquatiques comme les copépodes (DUSSARD, 1983) ont été effectuées en Guyane avant nos travaux sur le fleuve Sinnamary qui ont débuté en 1991 (HOREAU, 1996 ; RICHARD, 1996).

En janvier 1994, la mise en eau de la retenue hydroélectrique de Petit Saut a occasionné l'ennoiement de $365 \mathrm{~km}^{2}$ (HUYNH et al., 1996) de forêt primaire équatoriale située le long du fleuve Sinnamary dont le débit moyen est de $260 \mathrm{~m}^{3} \cdot \mathrm{s}^{-1}$. La hauteur maximale de la retenue est de 35 mètres et la hauteur moyenne de 11 mètres. L'évolution du milieu est essentiellement rythmée par les précipitations. En saison de pluies, le niveau de la retenue augmente malgré le turbinage important pour produire de l'énergie et atteint 35 mètres. En saison sèche, les débits turbinés sont plus importants que les débits entrants, entraînant la baisse du niveau de la retenue jusqu'à 29 mètres.

En Amazonie, de nombreux fleuves ont été aménagés. Les données hydrobiologiques disponibles sont peu nombreuses et concernent soit l'état initial du site soit son évolution après la perturbation. De plus, ces résultats sont souvent inaccessibles faute d'avoir été publiés dans des revues scientifiques et concernent uniquement la phase de remplissage ou des campagnes très ponctuelles (RochA et al., 1999). Néanmoins des études sur Brokopondo au Surinam (HEIDE, 1982) et sur quelques lacs brésiliens comme Samuel (FALOTICO, 1993) nous ont permis de comparer l'évolution des paramètres physico-chimiques et des peuplements d'invertébrés pélagiques durant la phase de remplissage. 
Sur l'aménagement de Petit Saut, l'acquisition de données régulières et continues sur de nombreux paramètres depuis la mise en eau de l'ouvrage a permis de mieux cerner les liens entre les paramètres abiotiques (pluviométrie, physico-chimie,...) et biotiques (phytoplancton, invertébrés aquatiques, poissons, ...) afin de mieux prévoir leur évolution. Ces informations et nos échanges constants avec l'exploitant permettent une meilleure gestion du barrage en vue de minimiser les impacts sur l'environnement.

\section{2 - MATÉRIELS ET MÉTHODES}

\subsection{Rythmicité et stations}

Dans la retenue, les évolutions saisonnière et inter-annuelle des paramètres physico-chimiques et des peuplements zooplanctoniques sont analysées à partir de relevés effectués chaque mois, dans la zone axiale de Roche Génipa (Génipa sur la figure 1).

Afin d'appréhender l'hétérogénéité spatiale de la retenue, quatre stations situées de l'axe du lac jusqu'à la berge, sur le transect de Roche Génipa au PK 23,6 (Génipa PK 23,6 sur la figure 1), sont échantillonnées deux fois par an, lors de la saison sèche et lors de la saison des pluies.

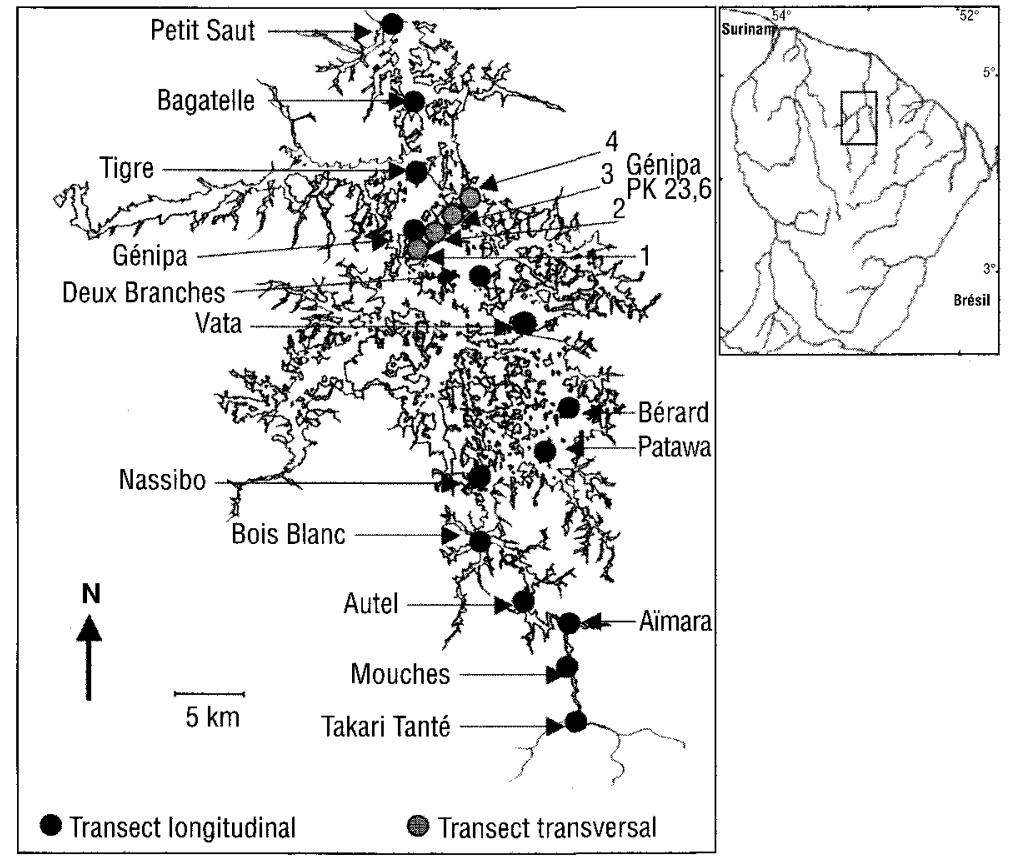

Figure 1 Localisation des stations.

Location of sampling stations. 
Enfin, des prélèvements de matériel biologique (phytoplancton et zooplancton) accompagnés de mesures d'oxygène dissous sont effectués selon un transect longitudinal qui comprend 14 stations situées de la queue de la retenue (Saut Takari Tanté) jusqu'en tête de retenue (Petit Saut) (figure 1).

\subsection{Paramètres et techniques de prélèvement}

Les précipitations relevées chaque jour sur le site du barrage ainsi que les débits entrants dans la retenue nous sont communiqués par l'exploitant (Électricité de France, EDF-Guyane).

Le temps de séjour mensuel de l'ensemble de la retenue est calculé en utilisant les débits entrants et le volume d'eau de la retenue, lui-même estimé par le niveau du lac (EDF-Guyane, comm. pers. ; SISSAKIAN, 1992).

De nombreux paramètres physico-chimiques sont mesurés dans le cadre du suivi de la qualité des eaux de la retenue mais nous n'utilisons pour cette présentation que des données obtenues à l'aide d'une multi-sonde YSI-GRANT qui fournit les paramètres suivants : oxygène dissous, température, $\mathrm{pH}$ et conductivité. Ces mesures sont effectuées tous les mètres de la surface à 10 mètres de profondeur puis tous les cinq mètres.

Les concentrations de chlorophylle et de bactério-chlorophylle sont effectuées par filtration d'eau prélevée aux mêmes niveaux que pour les paramètres précédemment cités. Des échantillons de $20-50 \mathrm{ml}$ sont filtrés sur membrane en fibre de verre Whatman GF/F (porosité de $0,7 \mu \mathrm{m}$ ), conservés au congélateur $\left(-20^{\circ} \mathrm{C}\right)$, puis dans l'azote liquide. Les pigments sont extraits en 24 heures au froid avec de l'acétone à $90 \%$, après broyage par sonication. Les dosages sont effectués suivant la méthode spectrofluorimètrique de NEVEUX et LANTOINE (1993), modifiée pour tenir compte des particularités du milieu.

En vue d'appréhender la répartition verticale du zooplancton dans la masse d'eau, 50 litres d'eau sont pompés à l'aide d'une pompe de type « fond de cale " ou d'une pompe électrique à immersion "LALIZAS' 1000LPH GPH 3800 ". L'eau est réceptionnée sur un filet de maille $50 \mu \mathrm{m}$ pour collecter les organismes. Ces pompages sont réalisés tous les mètres jusqu'à 10 mètres de profondeur, puis tous les 5 mètres jusqu'au fond.

Les comparaisons spatio-temporelles sont réalisées à partir de prélèvements verticaux. Les invertébrés sont alors collectés par traction verticale, depuis le fond jusqu'en surface, à l'aide de filets cylindro-coniques de $30 \mathrm{~cm}$ de diamètre d'ouverture et de $70 \mathrm{~cm}$ de hauteur. Le tissu filtrant est du nylon de $100 \mu \mathrm{m}$ de vide de maille. L'efficacité de ces traits de filet verticaux a été mise en évidence par BRANDL (1993). Au laboratoire, le comptage s'effectue, sous loupe binoculaire, sur un sous-échantillonnage, de quelques millilitres prélevés à la pipette (NIVAL, 1976) à la totalité du prélèvement, en fonction de la concentration du zooplancton. 6 à $100 \%$ de l'échantillon est alors examiné dans une cuve de Dolffus (PONT, 1977). Des écarts d'effectifs inférieurs à $5 \%$ ont été mesurés sur trois prélèvements identiques ainsi analysés.

Une analyse quantitative des profils verticaux montre que $95 \%$ de la biomasse des micro-crustacés se concentre dans la zone épilimnique (de la surface à un mètre au-dessous de l'oxycline) (HOREAU, 2004). La densité des micro-crustacés est donc rapportée au volume d'eau oxygéné +1 mètre. Les 
Chaoboridae occupant l'ensemble de la colonne d'eau, leur densité est rapportée au volume total prélevé.

Des coefficients permettent d'estimer la biomasse des invertébrés pélagiques. Les Chaoboridae ont fait l'objet de pesées selon la méthodologie utilisée par DUMONT et al. (1975) et MATSUMURA-TUNDISI et al. (1989). Trois séries de 20 individus ont été séchées et pesées sur une balance METLER AE 260. Malheureusement, les autres taxons du zooplancton n'ont pu être pesés sur ce type de balance présentant une précision à $10^{-4} \mathrm{mg}$ et les rapports tailles/poids de DUMONT et al. (1975) et de BOTTREL et al. (1976) se sont avérés inadaptés. Des estimations ont été réalisées en appliquant des règles de trois à partir des données de tailles et de poids secs de zooplancton du lac de Samuel (FALOTICO, 1993). Ces coefficients, présentés dans le tableau 1, permettent d'exprimer nos résultats en biomasse de poids $\sec \left(\mathrm{mg} / \mathrm{m}^{3}\right)$.

Dans le cadre du suivi hydrobiologique de la retenue de Petit Saut, les peuplements de poissons sont également étudiés. Leurs estomacs sont observés sur le terrain ou analysés sous loupe binoculaire au laboratoire, afin d'évaluer les aliments ingérés par la faune piscicole.

\section{3 - RÉSULTATS - DISCUSSION}

La mise en eau de Petit Saut a débuté le 6 janvier 1994. Le lac a atteint sa cote maximale un an et demi après, en juin 1995. Dès les premiers mois de la phase de remplissage, l'écosystème s'est comporté comme un lac. La masse d'eau se stratifie très rapidement, en un hypolimnion anoxique et un épilimnion oxygéné (RICHARD et al., 1997). Depuis le début du remplissage, l'épilimnion s'est épaissi pour passer de quelques centimètres en 1994 à 5-6 mètres à l'heure actuelle (figure 2). Les précipitations et la gestion de la production d'énergie déterminent le niveau de la retenue. Les pluies influent également sur le temps de séjour, paramètre reconnu très important dans le fonctionnement d'une retenue (SISSAKIAN, 1992 ; STRASKRABA, 1999). Sa moyenne est de six mois pour l'ensemble de la retenue, mais il varie de quelques jours à 16 mois selon les périodes (figure 3).

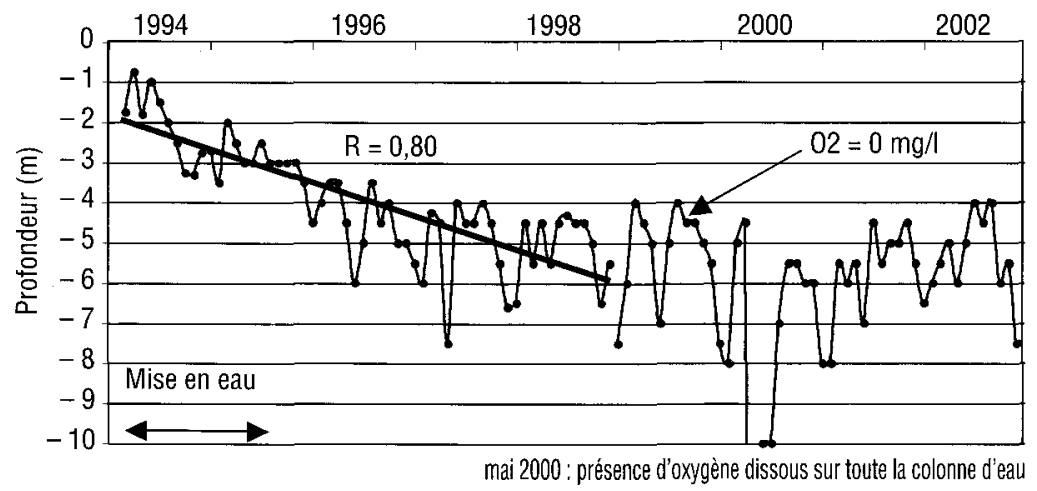

Figure 2 Oxycline de 1994 à 2002 à Roche Génipa.

Oxycline depth from 1994 to 2002 at Roche Génipa. 
Tableau 1 Invertébrés pélagiques collectés dans la retenue de Petit Saut (excepté les rotifères).

Table 1 Pelagic invertebrates collected in Petit Saut reservoir (except Rotifera).

\begin{tabular}{|c|c|c|c|c|c|c|}
\hline Classe & Famille & Taille (mm) & Poids sec $(\mu g)$ & Régime & Références & Espèce \\
\hline \multirow[t]{3}{*}{ Copépodes } & & & & & & \multirow{3}{*}{$\begin{array}{l}\text { Notodiaptomus echinatus (Lowndes, 1934) } \\
\text { Notodiaptomus n. sp. } \\
\text { Metacyclops hartmanni Herbst, 1960 } \\
\text { Thermocyclops minutus (Lowndes, 1934) } \\
\text { Mesocyclops venezolanus Dussart, 1987 } \\
\text { Mesocyclops meridianus (Kiefer, 1926) } \\
\text { Mesocyclops ellipticus Kiefer, } 1936\end{array}$} \\
\hline & Diaptomidae & 2,70 & 4,17 & Phyto - Rot. - Zoo & $A-B$ & \\
\hline & Cyclopidae & $0,39-1,11$ & 1,89 & Phyto - Bact. - Zoo & c & \\
\hline \multirow{3}{*}{$\begin{array}{l}\text { Cladocères } \\
\text { Sididae }\end{array}$} & & & & \multirow{3}{*}{$\begin{array}{l}\text { Phyto - Bact. } \\
\text { Phyto - Bact. } \\
\text { Petit Phyto - Bact. } \\
\text { Dét. - Petit Phyto - Bact. } \\
\text { Dét. } \\
\text { Dét. }\end{array}$} & \multirow{3}{*}{ D } & \\
\hline & & 0,58 & 1,84 & & & \multirow{2}{*}{$\begin{array}{l}\text { Diaphanosoma brevireme Sars, } 1901 \\
\text { Diaphanosoma sp. brevireme? } \\
\text { Ceriodaphnia cornuta Sars, } 1885 \\
\text { Bosminopsis deitersi Richard, } 1895 \\
\text { llyocryptus spinifer Herrick, } 1882 \\
\text { Leydigia sp. } \\
\text { Alona affinis (Leydig, 1860) } \\
\text { Kurzia latissima (Kurz, 1875) }\end{array}$} \\
\hline & $\begin{array}{l}\text { Daphniidae } \\
\text { Bosminidae } \\
\text { llyocryptidae } \\
\text { Chydoridae }\end{array}$ & $\begin{array}{c}0,25-0,38 \\
0,20\end{array}$ & $\begin{array}{c}1 \\
0,63 \\
2 \\
1\end{array}$ & & & \\
\hline \multirow{3}{*}{$\begin{array}{l}\text { Ostracodes } \\
\text { Conchostracés } \\
\text { Diptères }\end{array}$} & & & & & & \\
\hline & Candonidae & 0,48 & 1,89 & Dét. - Phyto - Bact. & E & $\begin{array}{l}\text { Physocypria affinis Klie, } 1933 \\
\text { Cyclestheria hislopi }\end{array}$ \\
\hline & Chaoboridae & $5-8$ & 40 & Phyto - Rot. - Zoo & $\mathrm{F}$ & Chaoborus sp \\
\hline
\end{tabular}

A - Pont, 1995 ; B - Pinel-Alloul, 1995a ; C - Soto \& Hurlbert, 1991 ; D - Burns, 1968 ; E - Martens, comm. pers. ; F - Pinel-Alloul, 1995b

Dét. : Détritus ; Bact. : Bactéries, Rot. : Rotifères ; Phyto : Phytoplancton ; Zoo : Zooplancton. 


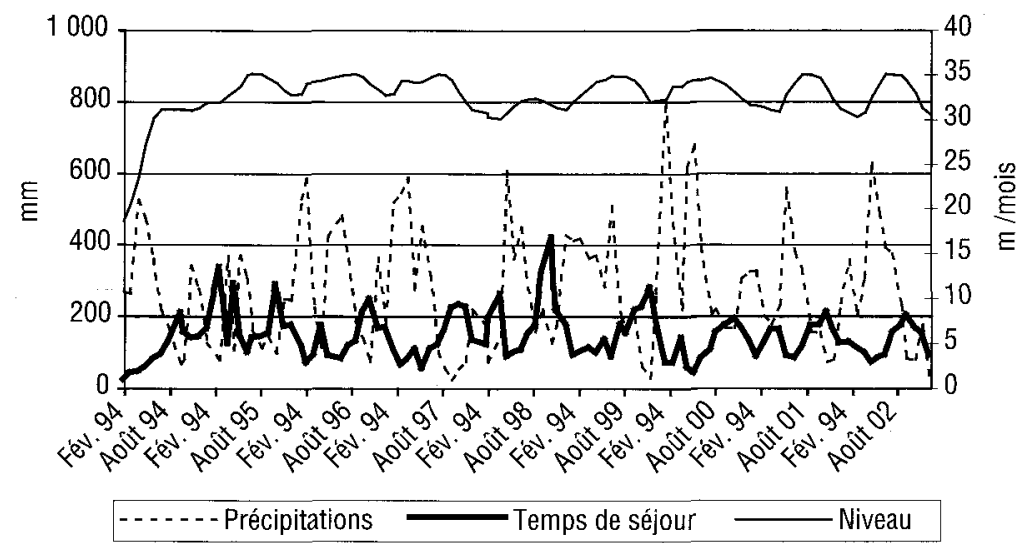

Figure 3 Niveau $(\mathrm{m})$, temps de séjour des eaux (mois) et précipitations $(\mathrm{mm})$ de 1994 à 2002.

Water level $(\mathrm{m})$, retention time (months) and precipitation $(\mathrm{mm})$ from 1994 to 2002.

Dès février 1994, un mois après le début de la fermeture des vannes, les invertébrés pélagiques sont abondamment représentés avec rotifères (Pourriot, 1996 ; PouRRIOT et al., 1997), cladocères, copépodes, ostracodes et larves de diptères Chaoboridae (tableau 1). Cette faune de milieu lentique dense et diversifiée colonise essentiellement l'épilimnion de la retenue. Effectivement, à part les Chaoboridae et quelques ostracodes, les invertébrés pélagiques se cantonnent encore dans la partie oxygénée (figure 4).

Contrairement à ce qui a été observé dans de nombreuses retenues amazoniennes (ARCIFA, 1984 ; ARCIFA et al., 1992 ; FALOTICO, 1993), durant la phase de remplissage, les rotifères sont peu denses dans la retenue de Petit Saut ( $<5 \%$ en nombre et $<1 \%$ en biomasse).

Les peuplements de cladocères sont essentiellement constitués de Bosminidae, de Sididae et de Daphniidae. Quelquefois, on note la présence de Chydoridae, et (ou) de llyocryptidae dans des biotopes tels que les racines des tapis flottants et les rives encore très boisées. Comme dans la majorité des retenues amazoniennes (ARCIFA 1984 ; HEIDE, 1982), Bosminopsis deitersi pour les Bosminidae et Ceriodaphnia cornuta pour les Daphniidae sont présents dans la retenue de Petit Saut. À l'inverse, pour les Sididae, Diaphanosoma brevireme n'est cité que dans le réservoir de Samuel (JUNK et al., 1981), le lac Manaquini (ROBERTSON et HARDY, 1984) et la rivière Orénoque (REY et VASQUEZ, 1989). Contrairement aux deux autres familles, aucune espèce de Sididae n'est ubiquiste des milieux amazoniens étudiés jusqu'à présent (HOREAU, 1996).

Les peuplements de copépodes sont constitués de cyclopoïdes et de calanoïdes (tableau 1). Thermocyclops minutus est une espèce de petite taille, ubiquiste des pays chauds qui s'étend dans toute la région tropicale et subtropicale de l'Amérique du Sud au nord de l'Argentine et au Venezuela. Ce copépode préfère des conditions oligotrophe et mésotrophe des grands lacs 
(REID, 1989). II est très bien représenté en Amazonie et est dominant en nombre dans la retenue de Petit Saut, durant la phase de remplissage (HOREAU, 1996). Une nouvelle espèce de calanoïde du genre Notodiaptomus est en cours de description.

La littérature permet d'effectuer des comparaisons de diversité avec d'autres lacs tropicaux amazoniens et africains. Néanmoins, elle reste fonction de l'âge des retenues ainsi que des investigations menées (prélèvements, taxonomistes, ...), c'est pourquoi ces comparaisons sont à prendre à titre indicatif. Dans la retenue de Petit Saut, on compte à l'heure actuelle 8 espèces de cladocères. Comparativement, le score maximum relevé dans les lacs tropicaux d'Amérique du Sud est de 23 taxons à Brokopondo au Surinam (HEIDE, 1982) et de 22 taxons à Segredo (RochA et al., 1999) pour le Brésil, le score minimum étant de 6 à Guarapiranga (DOMINGOS, 1993). Dans les lacs tropicaux africains, le nombre d'espèces de cladocères est inférieur à 8 (RochA et al, 1999). Dans la retenue de Petit Saut, on compte également 7 espèces de copépodes. Comparativement, la moyenne sur 10 lacs tropicaux brésiliens est de 8 avec un maximum de 15 à Segredo (ROCHA et al., 1999) et un minimum de 3 à L. Dourada (MELÃO, 1997). Le nombre d'espèces de copépodes varie entre 2 et 4 dans les lacs tropicaux africains (ROCHA et al., 1999).

En février 95, une population très nombreuse d'ostracodes est observée au site de Petit Saut (HOREAU et al., 1996). Ces organismes, habituellement benthiques, vivent en pleine eau dans cette retenue. Physocypria affinis Klie, 1933, très commun des Antilles est une espèce semi-planctonique, excellente nageuse (grâce aux longs poils natatoires sur les antennes) qui se trouve en grandes quantités dans la colonne d'eau, très loin du sédiment. Ceci n'est pas le cas pour la plupart des ostracodes qui, eux, sont benthiques (MARTENS, comm. pers.).
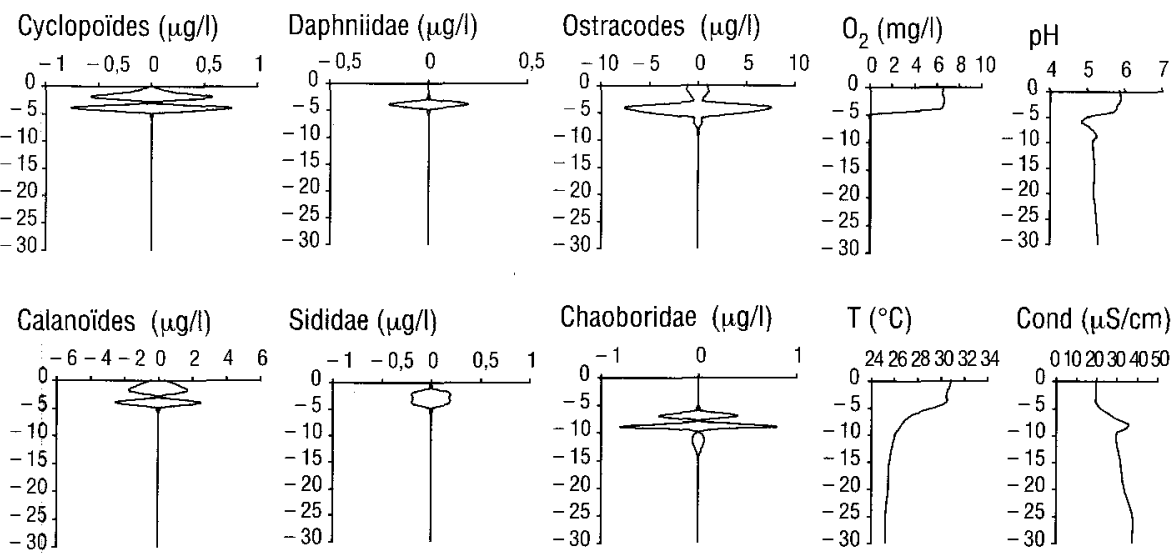

Figure 4 Répartition verticale de quelques paramètres physico-chimiques et des invertébrés pélagiques à Roche Génipa PK 23,6 (1), en septembre 2002.

Vertical distribution of physico-chemical parameters and pelagic invertebrates at Roche Génipa PK 23.6 (1) in September 2002. 
Les larves de Chaoboridae (Diptera) sont adaptées à la vie pélagique des lacs. Elles ont la possibilité remarquable de se réfugier un certain temps dans I'hypolimnion anoxique pour éviter la prédation des poissons (LAIR, 1995).

Depuis peu, des conchostracés sont observés de façon très sporadique dans la masse d'eau de Roche Génipa et plus souvent près des berges et dans des contenus stomacaux de poissons (VIGOUROUX, 2003). Cyclestheria hislopi est une espèce d'eaux semi-permanentes à permanentes, la plupart du temps parthénogénétique et courante dans la zone inter-tropicale (THIÉRY, comm. pers.).

\subsection{Répartition spatiale}

La retenue de Petit Saut n'est pas homogène et présente des différences de la tête de la retenue jusqu'à sa queue (répartition longitudinale) ainsi que de l'axe vers les berges (répartition transversale).

\subsubsection{Répartition longitudinale}

Durant la phase de remplissage, il se forme des gradients horizontaux du fait des masses d'eau qui possèdent une dynamique propre, en matière de circulation, de flux et de temps de renouvellement de l'eau. Il existe également un gradient dans la nature et dans la quantité des matériaux allochtones qui arrivent au lac. HEIDE (1982) à Brokopondo et TUNDISI (1988) au Brésil définissent trois zones plus ou moins distinctes: le fleuve, la zone intermédiaire ou de transition et le lac. À Petit Saut, l'hétérogénéité est mise en évidence par l'allure des courbes d'iso-concentrations en oxygène dissous (RICHARD, 1996 ; RICHARD et al., 1997). La masse d'eau de la zone de rivière est, comme la rivière, oxygénée de la surface jusqu'au fond avec des concentrations proches de la saturation, de l'ordre de 7-8 mg/l. La zone de transition évolue en fonction des saisons. Ainsi, en saison sèche, la station de Patawa présente une oxygénation jusqu'au fond avec des concentrations moins élevées que celles mesurées en rivière (figure 5). En saison des pluies, cette station appartient à la zone lacustre puisqu'elle est stratifiée en un épilimnion oxygéné et un hypolimnion anoxique, comme c'est le cas à Génipa. La distribution du phytoplancton et de la bactériochlorophylle distingue également trois zones géographiques qui diffèrent un peu de celles définies par l'oxygène dissous (VAQUER et al., 1997).

Les invertébrés aquatiques se distribuent également selon trois zones. On distingue ainsi la rivière avec notamment des Chironomidae et des Chydoridae qui restent très peu denses. La zone de transition étant oxygénée jusqu'au fond, les biomasses d'invertébrés rapportées au volume d'eau oxygénée restent faibles. Les éléments qui ont colonisé la retenue lors du remplissage y sont bien représentés, notamment les Cyclopoïdae. Cette zone s'étend jusqu'à Patawa en juin 1995 (hautes eaux) alors qu'elle s'arrête à Bois Blanc en décembre 2003 (basses eaux) (figure 6). La zone lacustre se caractérise par les Calanoïdes, les Sididae, les Ostracodes et les Chaoboridae. 

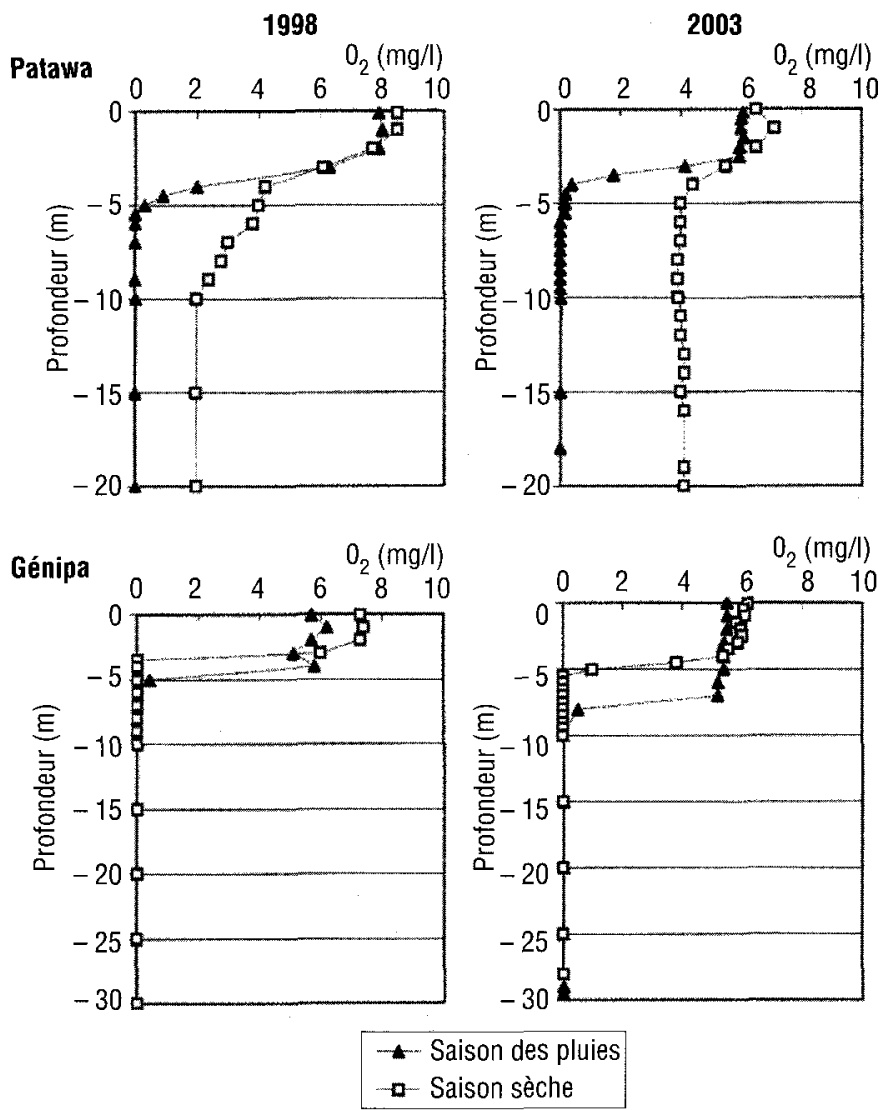

Figure 5 Oxygène dissous à Patawa et à Génipa en saison sèche et en saison des pluies des années 1998 et 2003.

Dissolved oxygen at Patawa and at Génipa during the dry and rainy seasons (1998 and 2003).
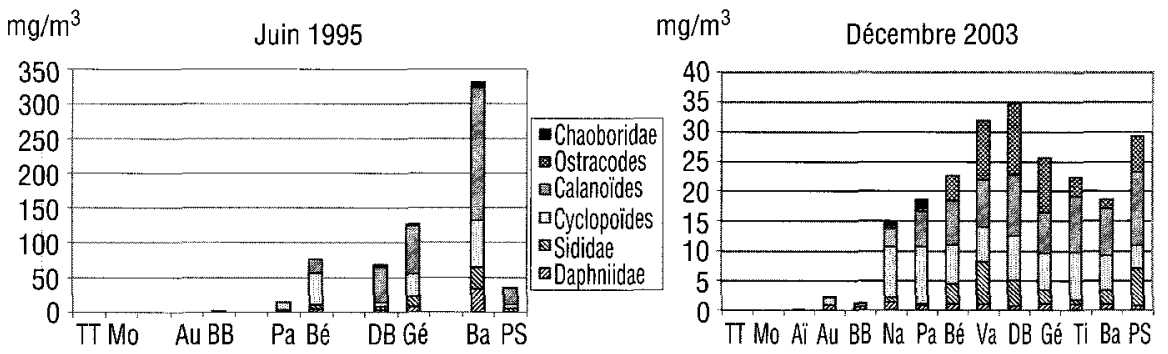

Figure 6 Répartition longitudinale des invertébrés pélagiques de la queue de la retenue ( $\Pi$ : Takari Tanté) jusqu'à sa tête (PS : Petit Saut), en saison des pluies 1995 et en saison sèche 2003.

Longitudinal distribution of pelagic invertebrates from the tail (TT) to the head (PS) of the reservoir during the rainy season (1995) and dry season (2003). 


\subsubsection{Répartition transversale}

La zone de forêt inondée représente un milieu plus encombré et plus riche en éléments dégradés que la zone centrale. De plus, du fait de l'encombrement, les masses d'eau ne circulent pas de la même façon que dans l'ancien axe du fleuve et le temps de renouvellement y est plus long. Ce biotope de forêt inondée occupe une superficie beaucoup plus importante dans la retenue de Petit Saut que la zone axiale correspondant aux anciens lits du fleuve et de ses affluents. De l'axe vers la berge, il existe un gradient des paramètres physico-chimiques, comme la conductivité (figure 7). De même, on note des concentrations de chlorophylle $a$ et de bactério-chlorophylle (VAQUER, comm. pers.) jusqu'à deux fois plus élevées dans les zones boisées que dans la zone axiale. En 1995, pendant la mise en eau, les plus fortes biomasses d'invertébrés aquatiques pélagiques étaient mesurées en zone axiale, probablement dues au fait que les zones boisées étaient à l'époque encore fermées par la végétation, ce qui excluait le développement du phytoplancton (HOREAU, 1996). Depuis, la végétation ennoyée a beaucoup évolué. II ne reste plus que des arbres morts sur lesquels persistent des épiphytes qui laissent passer la lumière jusqu'aux eaux permettant un développement phytoplanctonique. Chez les Chaoboridae, quelle que soit la saison, la biomasse de Chaoboridae est plus importante en berge que dans les autres stations. Pour le zooplancton, on met en évidence une différence selon les saisons. Ainsi, pendant la saison des pluies, les biomasses sont plus élevées dans la zone axiale que dans les autres stations avec toutefois des biomasses plus élevées en berge que dans les stations intermédiaires. En saison sèche, l'évolution globale est assez similaire si ce n'est que le maximum de biomasse est mesurée en berge (figure 8).

Les biomasses importantes obtenues en berge peuvent être expliquées par le fait que la matière organique ne se dégrade pas de la même manière en zone anoxique qu'en zone oxygénée. Ainsi dans la zone anoxique, la dégradation de la matière organique atteint un maximum pour donner du $\mathrm{CO}_{2}$ et du $\mathrm{CH}_{4}$, alors qu'en zone oxique, la dégradation n'est que partielle, ce qui fait un pool de matières carbonées utilisable par les organismes. La zone de forêt inondée est le siège d'une vie très intense due aux phénomènes de dégradation de la matière organique. Elle est plus riche en détritivores (Bosminidae, llyocryptidae et Ostracodes), en omnivores (Cylopoïdes) et en carnivores (Chaoboridae). À l'inverse, l'ancien lit du fleuve Sinnamary est moins densément peuplé et colonisé en majorité par des phyto-planctonophages (Calanoïdes et Daphniidae).

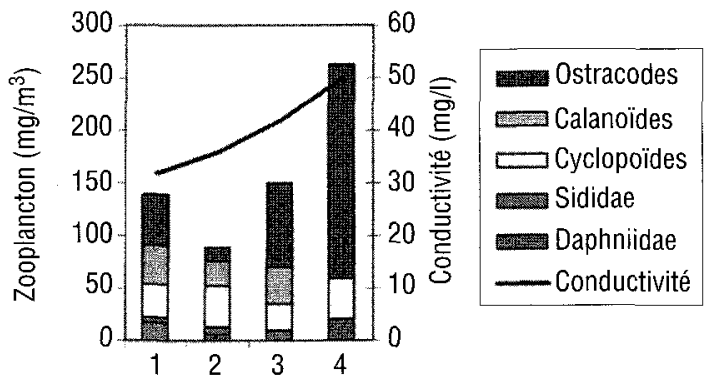

Figure 7 Répartition transversale de la conductivité et des invertébrés pélagiques de l'axe (1) vers la berge (4), en décembre 1996.

Distribution of conductivity and pelagic invertebrates from the axis of the reservoir (1) to its banks (4) in December 1996. 


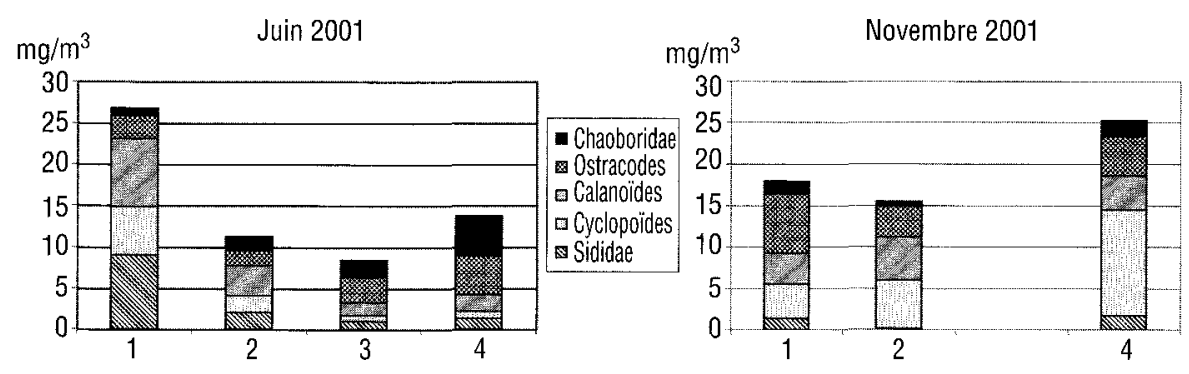

Figure 8 Répartition transversale des invertébrés pélagiques collectés de l'axe (1) vers la berge (4), en saison des pluies (juin) et en saison sèche (novembre) de l'année 2001.

Distribution of pelagic invertebrates from the axis of the reservoir (1) to its banks (4) during the rainy season (June) and in the dry season (November) 2001.

\section{2 Évolutions temporelles}

\subsection{1 Évolution inter-annuelle}

L'année 1994 ayant présenté un déficit hydrique, le remplissage de la retenue a nécessité deux saisons des pluies. À la fin de la première saison des pluies (juin 1994), la cote est de 31 mètres au site de Petit Saut. Elle atteint 35 mètres, la cote maximale souhaitée, en juin 1995. La colonisation a débuté par des peuplements de cladocères détritivores (Bosminidae) les quatre premiers mois. Dès mai 1994, les autres principaux cladocères (Daphniidae et Sididae) apparaissent dans le peuplement avec des copépodes (cyclopoïdes). Les Calanoïdes se développent en juin de la même année (HOREAU, 1996). Plus tard, vers la fin du remplissage, des ostracodes s'installent et sont très abondants jusqu'en 1997 (figure 9). Depuis 1998, leur contribution est inférieure à $5 \%$ de la biomasse des organismes pélagiques. La biomasse zoo-planctonique est très importante de 1994 à 1996 puis décroît jusqu'à la fin de l'année 2000, comme pour la biomasse piscicole (VIGOUROUX, 2003). On observe à nouveau une augmentation en 2001, probablement à mettre en relation avec une forte crue en mai 2000. Cette crue a fortement perturbé les peuplements et a permis la mobilisation de nouveaux nutriments. Ainsi, comme lors du remplissage de la retenue, de nombreuses niches écologiques ont été laissées vacantes et ont permis la re-colonisation et la prolifération des différents taxons.

Malgré leur poids relativement important, la biomasse des Chaoboridae est faible comparée à la biomasse des micro-crustacés. Ceci est lié au fait que cette biomasse est rapportée au volume contenu dans l'ensemble de la colonne d'eau. Leur évolution est assez similaire à celle du zooplancton si ce n'est que le pic de ces organismes a lieu en 1996-1997. Comme pour les micro-crustacés, on note un creux de biomasse de 1996 à 2000 qui pour les Choaboridae se poursuit jusqu'en 2002. Chez les copépodes, les fortes biomasses de calanoïdes mesurées au début du remplissage se retrouvent après la crue de 2000 . Chez les cladocères, les peuplements sont dominés par les Sididae depuis 1998. 


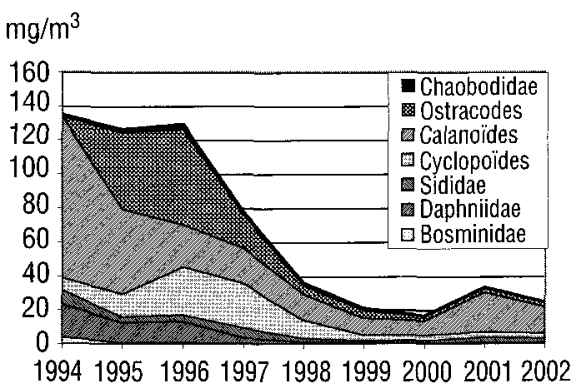

Figure 9 Biomasse des micro-crustacés et des Chaoboridae à Roche Génipa, de 1994 à 2002.

Biomass of micro-crustaceans and Chaoboridae at Roche Génipa from 1994 to 2002.

\subsection{2 Évolution saisonnière}

La diminution de la biomasse des micro-crustacés et des Chaoboridae peut être mise en relation avec la diminution de la conductivité qui globalise notamment les éléments nutritifs (figure 10). Effectivement, l'ampleur des pics des invertébrés aquatiques et de la conductivité diminue progressivement depuis le début du remplissage. Néanmoins, les plus fortes valeurs de conductivité sont obtenues en fin de saison sèche et les plus faibles en saison des pluies, liées aux phénomènes de dilution, alors que les creux de biomasse des invertébrés pélagiques sont obtenus en saison sèche. Ainsi, on assisterait à un appauvrissement des invertébrés pélagiques lié à une baisse des nutriments. Néanmoins, les pics de conductivité sont en inversion de phase avec les densités de zooplancton, ce qui s'explique par le fait que lorsqu'on est en présence des pics de nutriments, la masse d'eau est fortement stratifiée et ces éléments sont bloqués dans l'hypolimnion par l'oxycline. Lors de la saison des pluies, les apports en eau de l'amont de la retenue et les précipitations au-dessus de celle-ci, tendent à occasionner des petits mélanges entre les deux masses d'eau. Des nutriments sont alors disponibles dans la masse d'eau oxygénée et sont très rapidement assimilés jusqu'aux invertébrés.

La prédation par les poissons peut en partie expliquer certaines évolutions saisonnières de la faune des invertébrés aquatiques (figure 11). Effectivement, certains poissons profitent des apports exogènes lors de la saison des pluies: la faune aérienne est entraînée par les pluies et la faune qui se maintient sur les arbres entourés par les eaux lacustres est soumise à la montée des eaux. À l'inverse, lors de la saison sèche, certains poissons consomment du matériel endogène (essentiellement des micro-crustacés), comme c'est le cas de Triportheus rotundatus (HOREAU et al., 1998). Globalement, de 1994 à 2002, la contribution des invertébrés aquatiques est plus importante en saison sèche (36 \%) qu'en saison des pluies (25\%). II faut surtout souligner la contribution des micro-crustacés qui est de $13 \%$ en saison sèche contre $6 \%$ en saison des pluies. 


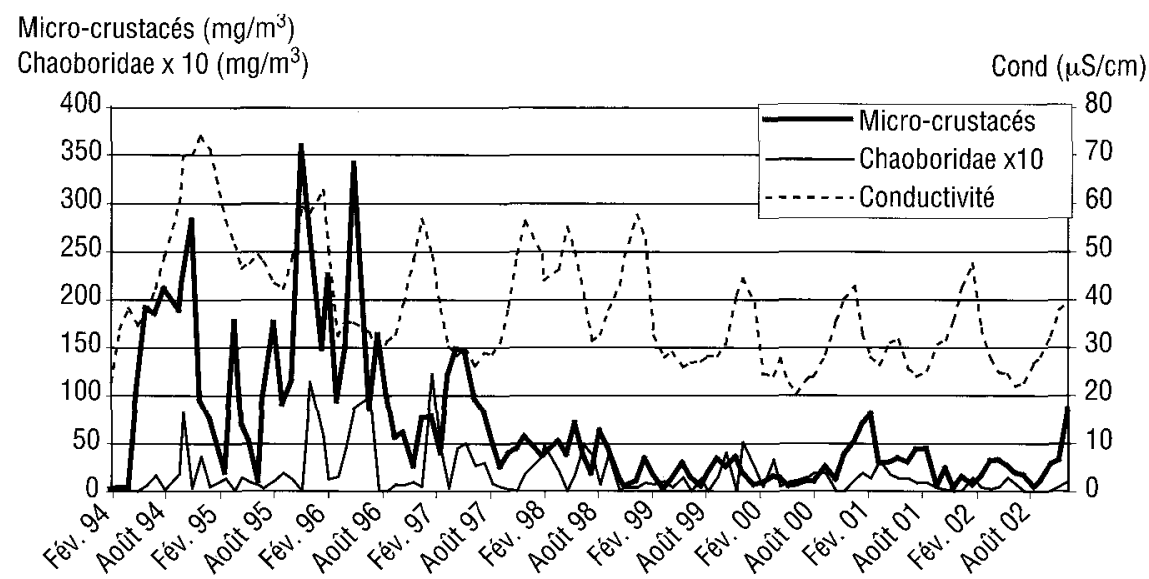

Figure 10 Invertébrés pélagiques et conductivité moyenne à Roche Génipa de 1994 à 2002.

Pelagic invertebrates and average conductivity at Roche Génipa from 1994 to 2002
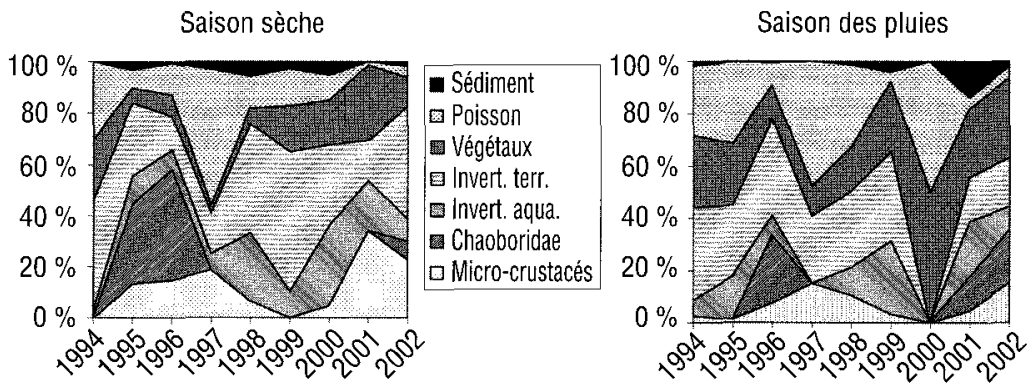

Figure 11 Nourriture des poissons de la retenue, de 1994 à 2002 en fonction des saisons.

Fish stomach contents from 1994 to 2002 in accordance with seasons.

\section{4 - CONCLUSIONS}

Le réservoir hydroélectrique de Petit Saut, mis en eau en janvier 1994 a occasionné la mise en place d'un écosystème stratifié. La dégradation de la litière et des feuilles des $365 \mathrm{~km}^{2}$ de forêt ennoyée a très rapidement consommé l'oxygène dissous de la masse d'eau créant un milieu stratifié en un épilimnion oxygéné de quelques centimètres et un hypolimnion anoxique. L'hypolimnion est anoxique et riche en éléments réduits. Les éléments nutritifs accumulés au fond de la retenue diminuent progressivement, oscillant aux rythmes des saisons; en saison sèche, ces éléments se concentrent et inver- 
sement, en saison des pluies, ils sont dilués par les apports en eau sur le bassin versant. Aussi, assiste-t-on à une amélioration progressive de la qualité physico-chimique des eaux de la retenue.

La faune de rivière a disparu et a été remplacée en quelques semaines par du zooplancton diversifié, cantonnée à l'épilimnion excepté pour les Chaoboridae. D'abord dominés par des détritivores (Bosminidae) et des Cyclopoïdes, les peuplements se sont diversifiés avec des phytophages (Calanoïdes et Daphniidae) lorsque le phytoplancton s'est développé; puis des carnassiers, Chaoboridae. Ce cortège est rapidement accompagné par des Sididae et des ostracodes.

La retenue n'est pas homogène et présente une zonation longitudinale mise en évidence par les iso-concentrations en oxygène dissous, les quantités de phytoplancton et de bactério-chlorophylles ainsi que par les quantités et la qualité du zooplancton. De même, on met en évidence un gradient croissant de la conductivité pour une même profondeur de l'axe à la berge qui occasionne de plus fortes densités d'invertébrés aquatiques près des berges en saison sèche. Très élevées lors du remplissage, liées aux grandes quantités de matières organiques disponibles, les biomasses décroissent progressivement en relation avec la baisse des nutriments. Ces organismes jouent un rôle fondamental dans le fonctionnement d'un tel écosystème notamment en consommant la production primaire et en étant consommés par les maillons supérieurs comme les poissons.

Âgée de dix ans, la retenue voit encore se développer de nouveaux taxons comme c'est le cas du conchostracé Cyclestheria hislopi que l'on observe depuis début 2003. Cette apparition témoigne de l'évolution encore actuelle du système lacustre.

\section{REMERCIEMENTS}

Cette étude a été financée par EDF dans le cadre d'un contrat HYDRECO/EDF-Guyane. Nous tenons à remercier toutes les personnes qui y ont contribué, notamment les spécialistes qui ont déterminé le matériel, D. Defaye, K. Martens, R. Pourriot, J.C. Paggi et A. Thiéry ainsi qu'Andréa Dejean et Sylvie Jérémie pour les traductions anglaises et l'équipe technique du Laboratoire Environnement de Petit Saut, R. Aboïkoni, B. Burban et C. Reynouard. 


\section{RÉFÉRENCES BIBLIOGRAPHIQUES}

ARCIFA M. S., 1984. Zooplankton composition of ten reservoirs in southern Brazil. Hydrobiologia, 113, 137-145.

ARCIFA M. S., GOMES E. A. T., MESCHIATTI A. J., 1992. Composition and fluctuations of the zooplankton of tropical brazilian reservoir. Arch. Hydrobiol, $123,479-495$.

BOTTREL H.T., DUCAN A., GLIWICZ Z., GRYGIEREK E., HERZIG A., HILLBRIGHT-ILKOWSKA A., KIRASAWA H., LARSSON P., WEGLENSKA T., 1976. A review of some problems in zooplankton production studies. Norw. J. Zool., 24, 419-456.

BRANDL Z., 1993. Precision of net zooplankton sampling from reservoirs by vertical hauls. Arch. Hydrobiol. Beih. Ergebn. Limnol., 40, 155-159.

BURNS C. W., 1968. The relationship between bodysize of filter feeding Cladocera and the maximum size of particle ingested. Limnol. Oceanogr., 13, 675678.

DOMINGOS M. D., 1993. Spatial Heterogeneity (horizontal) of Guarapiranga Reservoir (São Paulo) and the Distribution of Zoopiankton Community. Ph. D. Thesis, São Paulo, Brésil, 156 p.

DUMONT H.J., VELDEI (VAN DE), DUMONT S., 1975. The dry weight estimate of biomass in a selection of Cladocera, Copepoda and Rotifera from the plankton, periphyton and benthos of continental waters. Oecologia, 19, 75-97.

DUSSARD B., 1983. Copépodes d'eau douce de Guyane française. Rev. Hydrobiol. Trop., 16, 321-325.

FALOTICO M.H.B., 1993. Características timnológicas e aspectos da composição e distribuição da comunidade zooplanctônica em sua fase de enchimento (reservatório de Samuel - Rondônia). Msc. Thesis USP, São Paulo, Brésil, 195 p.

HEIDE (VAN DER) J., 1982. Filling phase limnology of man-made lake in the humid tropics. Lake Brokopondo, Offsetdrukkerij Kanters B.V., Alblasserdam.

HOREAU V., 1996. La mise eau de la retenue hydroélectrique de Petit Saut (Guyane).
Hydrobiologie 1- du fleuve Sinnamary avant la mise en eau, 2- de la retenue pendant la mise en eau, 3- du fleuve en aval. Th Doct. Univ. Aix-Marseille I, France, $250 \mathrm{p}$.

HOREAU V., CHAMPEAU A., GREGOIRE A., CERDAN P., 1996. Hydrological aspects of impouding at Petit Saut (French Guiana). Comptes rendus Symposium International d'Ecohydraulique 2000. Canada, Québec, juin 1996, vol. A, 3748.

HOREAU V., RICHARD S., CERDAN P., 1998. La qualité de l'eau et son incidence sur la biodiversité. L'exemple de la retenue de Petit Saut (Guyane Française). JATBA, 40, 53-77.

HOREAU V., 2004. Les invertébrés aquatiques de la retenue de Petit Saut (Guyane) et du tronçon aval. HYDRECO/EDF-CD-Guyane, Petit Saut, Guyane française, France, Rapport scientifique, $46 \mathrm{p}$.

HUYNH F., DEMAGISTRI L., CHARRON C., PANECHOU K., GARDEL A., HERNANDEZ L., LOUBRY D., 1996. Cartographies de la retenue du barrage de Petit Saut aux cotes 31 et 35 mètres. ORSTOM-EDF, Cayenne, Guyane Française, France, Rapport scientifique, $64 \mathrm{p}$.

JUNK W. J., ROBERTSON B. A., DARWICH A. J., VEIRA I., 1981. Investigaçoes limnologicas e ictiologicas em Curua-Una, a primeira represa hidrelética na Amazôniana Central. Acta Amazonica, 11, 689-716.

LAIR N., 1995. Croissance et dynamique des populations d'invertébrés planctoniques. In "Limnologie Générale Collection d'écologie 25 ", Pourriot R. et Meybeck M. [Ed.], pp. 351-367.

MATSUMURA-TUNDISI T., TUNDISI J.G., SAGGIO A., OLIVEIRA NETO A.L., ESPINDOLA E.G., 1989. Limnology of Samuek Reservoir (Brasil, Rondônia) in the filling phase. Verh. Internat. Verein. Limnol., 1482-1488.

MELÃO M. G. M., 1997. Plankton community (phytoplankton and zooplakton) and secondary productivity in a oligotrophic reservoir. Ph. D. Thesis, Fed. Univ. São Carlos, Brésil, 258 p. 
NEVEUX J., LANTOINE F., 1993. Spectrofluorometric assay of chlorophylls and phaeopigments using the least squares approximation technique. Deep-Sea Research, 40, 1747-1765.

NIVAL P., 1976. Relation zooplancton-phytoplancton. Essai de modélisation. Th. Doct. Univ. P. \& M. Curie, Villefranchesur-Mer, France, $219 \mathrm{p}$.

PINEL-ALLOUL B., 1995a. Les invertébrés prédateurs du zooplancton. In "Limnologie Générale Collection d'écologie 25 », Pourriot R. et Meybeck M. [Ed.], pp. 541-564.

PINEL-ALLOUL B., 1995b. Impacts des prédateurs invertébrés sur les communautés aquatiques. In " Limnologie Générale Collection d'écologie 25 ", Pourriot R. et Meybeck M. [Ed.], pp. 628-647.

PONT D., 1977. Recherches sur l'évolution saisonnière du peuplement des copépodes, cladocères et ostracodes des rizières de Camargue. Th. Doct. Univ. Marseille, France, $110 \mathrm{p}$.

PONT D., 1995. Le zooplancton herbivore dans les chaînes alimentaires pélagiques. In "Limnologie Générale Collection d'écologie 25 », Pourriot R. et Meybeck M. [Ed.], pp. 515-540.

POURRIOT R., 1996. Rotifers from Petit Saut reservoir (French Guyana), with the description of a new taxon. Hydrobiologia, 331, 43-52.

POURRIOT R., HOREAU V., ROUGIER C., 1997. Filinia (Rotifera, Monogononta) populations in french Guyana: F. novaezealandiae, $F$. saltator ovilli remarks on the variability of some characters. Arch. Hydrobiol., 139, 563-575.

REID J. W., 1989. The distribution of species of the genus Thermocyclops (Copepoda, Cyclopoida) in the Western Hemisphere, with description of $T$. parvus, new scies. Hydrobiologia, 175, 149-174.

REY J., VASQUEZ E., 1989. Bosminopsis brandorffi n. sp. (Crustacea, Cladocera) une nouvelle espèce de Bosminidae des systèmes Amazone et Orénoque. Annals Limnol., 25, 215-218.

RICHARD S., 1996. La mise eau de la retenue hydroélectrique de Petit Saut (Guyane). Physico-chimie 1- du fleuve Sinnamary avant la mise en eau, 2- de la retenue pendant la mise en eau, 3- du fleuve en aval. Th. Doct. Univ. Aix-Marseille I, France, 278 p.

RICHARD S., ARNOUX A., CERDAN P., 1997. Évolution de la qualité physicochimique de l'eau de la retenue et du tronçon aval depuis le début de la mise en eau du barrage de Petit Saut. Hydroécol. Appl. 9, 57-83.

ROBERTSON B. A., HARDY E. R., 1984. ZoOplankton of amazonienne lakes and rivers. In "The Amazon. Limnology and landscape ecology of mighty tropical river and its bassin ", Sioli H. [Ed.], pp 337-352.

ROCHA O., MATSUMURA-TUNDISI T., ESPINDOLA E. L. G., ROCHE K. F., RIETZLER A. C., 1999. Ecological theory applied to reservoir zooplankton. In "Theoretical Reservoir Ecology And its Applications ", Tundisi J.G. and Straskraba M. [Ed.], pp. 457-476.

SISSAKIAN C., 1992. Présentation de la retenue de Petit Saut en Guyane française : cartographie - partition de la retenue volumes et surfaces - intégration paysagère. Hydroécol. Appl., 4, 213-240.

SOTO D., HURLBERT S. H., 1991. Short term experiments on calanoid-cyclopoid-phytoplankton interactions. Hydrobiologia, 215, 83-110.

STRASKRABA M., 1999. Retention time as a key variable of reservoir limnology. In "Theoretical Reservoir Ecology And Its Applications ", Tundisi J.G. and Straskraba M. [Ed.], pp. 385-410.

TUNDISI J. G., 1988. Limnologia e manejo de represas. EESC-SP/CHREA/ACIESP, São Carlos, Brésil, Rapport scientifique, $506 \mathrm{p}$.

VAQUER A., PONS V., LAUTIER J., 1997. Distribution spatio-temporelle du phytoplancton dans le réservoir de Petit Saut (Guyane). Hydroécol. Appl., 9, 169-193.

VIGOUROUX R., 2003. Suivi écologique de la retenue de Petit Saut et du fleuve Sinnamary à l'aval du barrage. Ichtyologie : récapitulatif et analyse des données obtenues depuis le début du suivi du site. Évolution des peuplements de poissons depuis la mise en eau du barrage ; influence des événements hydrologiques et climatiques ; analyse détaillée des campagnes de 2001 - 2002 ; perspectives d'évolution. HYDRECO-EDF Guyane, Petit Saut, Guyane française, France, Rapport scientifique, $50 \mathrm{p}$. 